\title{
The application of Virtualization technology in Grid Computing
}

\author{
Zhang Cheng ${ }^{1, ~ a ~}$, He Dan ${ }^{2, b}$, XiaoJianlian $^{1, \mathrm{c}}$, LiYang $^{1, \mathrm{~d}}$ \\ ${ }^{1}$ City Institute, Dalian University of Technology, Dalian Liaoning 116600, China \\ ${ }^{2}$ Dalian University of Finance and Economics, Dalian Liaoning 116622, China \\ a13130493795@wo.com.cn, ${ }^{\mathrm{a}}$ kerrychinaren@live.cn, ${ }^{\mathrm{c} x j l @ q q . c o m,{ }^{\mathrm{d}} l y @ d l u t . e d u . c n}$
}

Keywords: Grid computation; Virtual machine; Virtual network; Grid

\begin{abstract}
In this paper, the author discussed the current popular the grid computation technique and the virtualization technology, analyzed and concluded characteristic of two technologies. Then, VNET has been proposed, which can add the virtual machine to the network efficiently. It can dynamically modify its topology and routing rules on load situation of the network flows. At last, a virtual machine was setup on parallels work station and the related experiments were taken with satisfied results.
\end{abstract}

\section{Introduction about virtualization technology}

Virtual computer.With special software and hardware technologies,the virtual computer split the host computer in many "virtual” host ones[1], each which has an independent domain name and IP address (or a Shared IP address) and own a complete servicefunction of Internet.

The classification of the virtual computer.A typical computer has three components[2]: hardware, operating systems, applications.And there are two main interfacesin the computer, they are respectively referred to as ISA (instruction set interface), ABI (application binary interface).As shown in figure 1.



(a) ISA

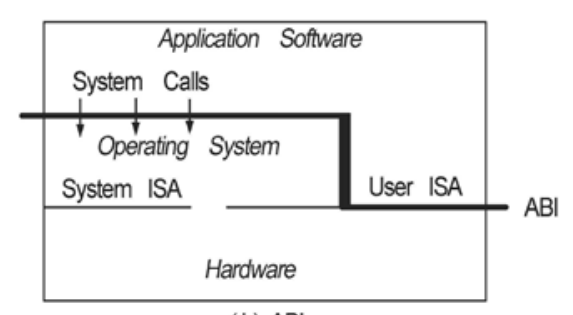

(b) $\mathrm{ABI}$

Fig. 1 classification of the virtual machine

As seen from figure 1, the instruction set interfaces include the ISA of the user sets and the system sets. The ISA of the user sets are used by the operating system and applications. The ISA of the system sets can only be used by the operating system and only the privilege set of instructions can be used to directly manipulate the processor, memory, IO. As seen from the figure 1 (b) the application binary interface includes the ISA of the user sets and the system calls. There is system call interface between application software and the operating system, which is used to protect and management the hardware resources.

Virtual machine located in the operating system layer and Virtual Machine Management (VMM). The virtual machine located in the operating system layer has been classified as the virtual machine with ISA (the instruction set interface)and there can be same execution environment as the ones in the whole operating system[3]. The starting purpose by the introduction of the virtual machine located in the operating system layer is to use the expensive hardware resources together by the abstraction of hardware resources. The abstraction layer of hardware resources is called virtual machine management (VMM), which can run different operating system on the same hardware platform. 


\section{Grid computing}

The introduction of grid computing.Grid computing that is a new calculation model for complex scientific computing is rapidly developing with the internet technology[4]. The calculation mode can organize the computers distributed in different places into a "virtual supercomputer", in which each computer participating in the calculation is a node and the whole calculation is a grid made up of tens of thousands of nodes. So the calculation is called grid computing. Such organized virtual supercomputer has two advantages, one is the strong data processing ability; Another is to make full use of idle processing capacity in the Internet. Simply put, the grid integrates the whole network into a huge supercomputer to implement the full shared resources such as calculation, storage, data, information, knowledge and experts.

Gird computing with the participation of the virtual machine located in the operating system layer[5].Gird computing with the participation of the virtual machine located in the operating system layer consists of a real mirror image, a virtual OS mirror image, a mirror image of the application layer and a user data server. The computing environment is shown in figure 2.

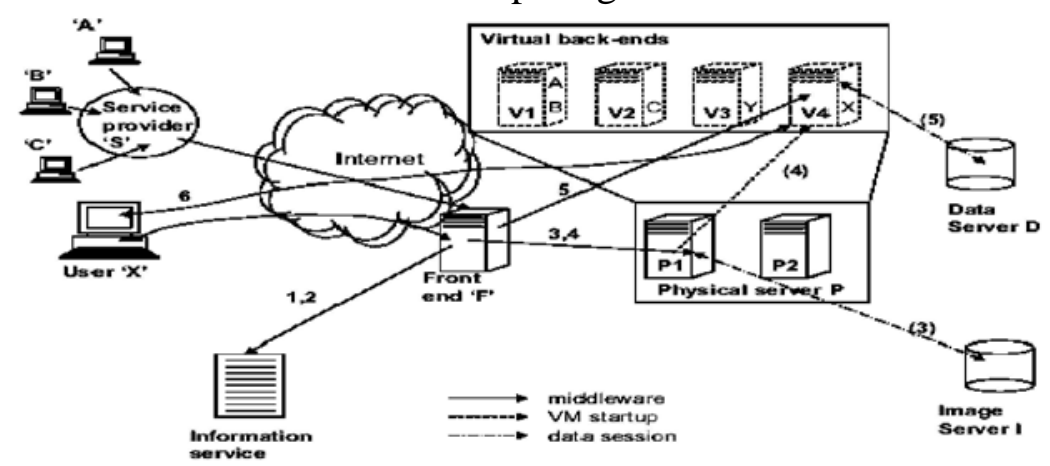

Fig.2Architecture of grid computing in a virtual machine

According to figure 2, when the user $\mathrm{X}$ through the information service inquires the resources available, such as GlobusMDS, the data session is established between physical server and mirror server of the grid middleware. When the data session is established, the former from the mirror server downloads the mirror image and after completing the above a part of resources is distributed into the mirror image of the virtual machine by a physical machine and then the virtual machine has been initialized by the distribution of IP and the MAC address.Virtual backend is a set of virtual machines, they are mapped to the part of the physical machine, and then the data session is established between machine operating system and the application server on the virtual machine. The application is downloaded.

The management of virtual network. As can be seen from the figure 3, the architecture has three layers[6]: one is the layer of their own of the virtual machine;The other is the layer of the virtual machine background (VMD); The last isthe physical layer where VMD is running on. The layer of VMD is the general expression of virtual network server and it can be used to manage the virtual machine and measure the traffic and its nature of low-levelnetwork.Nodes and edges of virtual layer are mapped to the point and path of thelayer of VMD.The physical layer is actually IP network itself. Nodes of the layer of VMD are mapped to the terminal system of the network.

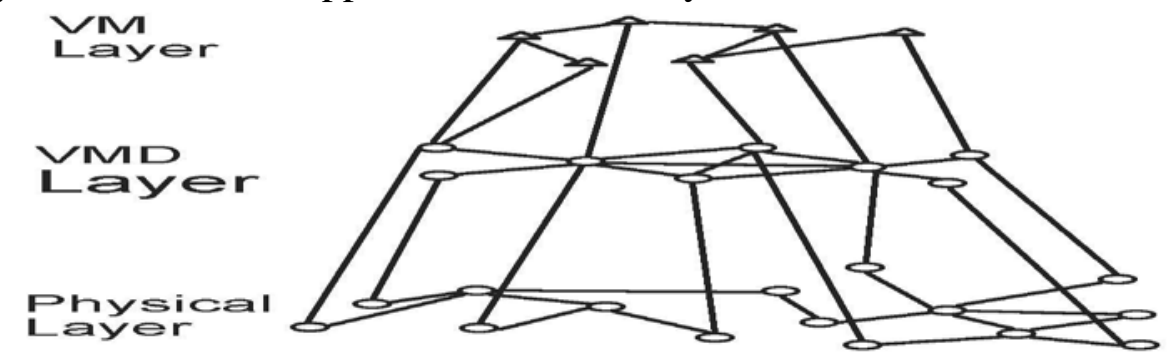

Fig.3 the virtual network architecture

The layer of VM contains the virtual machine and edges between them, and the layer of VMDincludes the background program of the virtual machine and edges between them . If the virtual machine $\mathrm{Vi}$ sends information to $\mathrm{Vj}$, there must be a routing from the $\mathrm{Vi}$ to $\mathrm{Vj}$.Each virtual 
machine must be assigned to the layer of VMD where it can maintain some parameters such asnetwork bandwidth, delay and computing capacity of every nodes etc. The physical layer contains some low-level topological structure, and each node of VMD layer is mapped to the physical layer where there has been at most onenode of VMD mapped in each host.

Support random topology and routing. There are connections through TCP between each node in the layer of VMD. Such is the setting that the user may establish a network topological structure of the star type around the center of the agent server. If so all the information must go through the agent server.It can be clearly seen that supporting random topological structure is possible because the VMD can work like switches or routers and will send a packet from a TCP connection to another TCP connections, rather than to the local area network (LAN). Indeed we can simulate its exchange protocol for Ethernet such as the VIO - LIN. Each packet contains a source and destination addresses. A switch for modern Ethernet can see the position of the port according to its source address and run a distributed algorithm on the switch. So the topological structure of the tree type is formed.

\section{Creating VM on the parallels platform}

Parallels is a VMM and it supports to create any number of virtual machines and the latest Internet virtualization technology. Experimenting on its platform, we chose the GlobusToolkits5 that is a middleware of grid computing environment constructed and provides the basic services such as resources orientation, management, communication and security etc. The calculation toolkit is modularized and the users are allowed to customize the environment according to their own need. This set of tool can be used to establish computing grid and undertake the development of grid application.Environment is built with two machines : two virtual machines run on one machine and the other is common machine whose computing tasks is to generate Mandelbrot set. The experimental results is shown in figure 4.



Fig.4 experimental results

\section{Conclusions}

Virtual machine is very flexible and it can be created randomly and initialized dynamically. These features used in grid computing can support transfer of the computing environment, management of customer data, separation and good security. When the virtual machine is applied to grid computing, the biggest challenge is how to overcome the performance degradation. Technology stated in the article can improve performance and avoid the shortcoming of full virtualization in the grid computing. 


\section{References}

[1]YaqiongLi, YingSong,YongbingHuang.A Memory Global Optimization Approach in Virtualized CloudComputing Environments [J].CHINESE JOURNA L OF COMPU TERS, 2011(4):684-689.

[2]HaiJin, Xiaofei Liao.Virtualization Technology for Computing System[J].China Ba sic Science,2008(6) :11-18.

[3]KejiangYe,ZhaohuiWu,XiaohongJiang,Qingming He. Power Management of Virtualized Cloud Computing Platform[J].CHINESE JOURNA L OF COMPU TERS, 2012(4):1262-1270.

[4]ShuangquanLi, YanweiWang.Research on the Server Virtualization Technology in Cloud Computing[J].Designing Techniques of Posts and Telecommunications,2011(10):27-32.

[5]Nianqiang Zhao,ShiguangJu. Survey of grid computing and grid architecture[J]. Computer Engineering and Design,2006(3):728-734.

[6]Kim Y, Perrig A, Tsudik G. Tree-based group key agreement[J].ACM Transactions on Information and System Security (TISSEC),2004, 7(1):60-96. 